\title{
The role of population PK-PD modelling in paediatric clinical research
}

\author{
Roosmarijn F. W. De Cock • Chiara Piana • \\ Elke H. J. Krekels • Meindert Danhof • \\ Karel Allegaert • Catherijne A. J. Knibbe
}

Received: 9 July 2009 /Accepted: 22 December 2009/Published online: 26 March 2010

(C) The Author(s) 2010. This article is published with open access at Springerlink.com

\begin{abstract}
Children differ from adults in their response to drugs. While this may be the result of changes in dose exposure (pharmacokinetics $[\mathrm{PK}]$ ) and/or exposure response (pharmacodynamics $[\mathrm{PD}]$ ) relationships, the magnitude of these changes may not be solely reflected by differences in body weight. As a consequence, dosing recommendations empirically derived from adults dosing regimens using linear extrapolations based on body weight, can result in therapeutic failure, occurrence of adverse effect or even fatalities. In order to define rational, patienttailored dosing schemes, population PK-PD studies in children are needed. For the analysis of the data, population modelling using non-linear mixed effect modelling is the preferred tool since this approach allows for the analysis of sparse and unbalanced datasets. Additionally, it permits the
\end{abstract}

Roosmarijn F. W. De Cock and Chiara Piana contributed equally to this article

R. F. W. De Cock $\cdot$ C. Piana E. H. J. Krekels $\cdot$ M. Danhof $\cdot$

C. A. J. Knibbe

Division of Pharmacology,

Leiden/Amsterdam Centre for Drug Research, Leiden University,

Leiden, The Netherlands

E. H. J. Krekels • C. A. J. Knibbe

Department of Pediatric Intensive Care,

Erasmus MC-Sophia Children's Hospital,

Rotterdam, The Netherlands

C. A. J. Knibbe $(\square)$

Department of Clinical Pharmacy, St. Antonius Hospital, P.O. Box 2500, 3430 EM Nieuwegein, The Netherlands e-mail: c.knibbe@antoniusziekenhuis.nl

K. Allegaert

Neonatal Intensive Care Unit, University Hospital Leuven, Leuven, Belgium exploration of the influence of different covariates such as body weight and age to explain the variability in drug response. Finally, using this approach, these PK-PD studies can be designed in the most efficient manner in order to obtain the maximum information on the PK-PD parameters with the highest precision. Once a population PK-PD model is developed, internal and external validations should be performed. If the model performs well in these validation procedures, model simulations can be used to define a dosing regimen, which in turn needs to be tested and challenged in a prospective clinical trial. This methodology will improve the efficacy/safety balance of dosing guidelines, which will be of benefit to the individual child.

Keywords Pharmacokinetics · Pharmacodynamics · Population modelling $\cdot$ Paediatric population · Validation

\section{Introduction}

Children differ from adults in their response to drugs. These differences may be caused by changes in the pharmacokinetics (PK) and/or pharmacodynamics (PD) between children and adults and may also vary among children of different ages. The PK of a drug includes the processes of absorption, distribution, metabolism and elimination of a drug, whereas the PD comprises the physiological and biological response to the administered drug and therefore may represent both efficacy and safety measures. While a child grows, enzyme pathways (involved in the PK), function and expression of receptors and proteins (involved in the PD) mature, which can be referred to as "developmental changes" in childhood. The maturation rates of these developmental changes vary, however, between the pathways and receptors and often do not correlate solely with 
the increase in body weight of the child. The question is therefore how to obtain data in children that allow for the study of these developmental changes, ultimately resulting in evidence-based dosing regimens for drugs in children.

To date, only a small number of drugs used in children are licensed for use in this specific group. Up to $70 \%$ of the drugs in paediatric intensive care, and $90 \%$ of the drugs in neonatal intensive care, are prescribed in an off-label or unlicensed manner [1-4]. Paediatric dosing regimens are usually empirically derived from adult regimens using linear extrapolations based on body weight. Since these developmental changes are non-linear dynamic processes, this dosing paradigm may result in under- or over-dosing, particularly in specific age groups. This may cause therapeutic failure, occurrence of severe adverse effects or even fatalities, such as fatalities occurring after long-term sedation with high doses of propofol $[5,6]$ and occurrence of the grey baby syndrome in neonates after treatment with chloramphenicol $[7,8]$. As a result, dose adjustments in the younger age groups are often proposed. For vancomycin, for example, lower doses are administered in neonates younger than 1 week $(20 \mathrm{mg} / \mathrm{kg} /$ day) compared with 1 - to 4-week-old neonates $(30 \mathrm{mg} / \mathrm{kg} /$ day $)$ and children between 1 month and 18 years $(40 \mathrm{mg} / \mathrm{kg} /$ day) [9].

Instead of the a priori use of body weight for dosing guidelines in children, detailed information on PK and potentially also the PD needs to be considered in order to define effective and safe dosing regimens throughout the paediatric age range. The lack of PK and PD information on drugs in children has led to the European Regulation, which came into force in 2007. This law imposes on pharmaceutical companies to perform research over the whole paediatric age range for all drugs that are developed for the European market, by requiring the submission of a paediatric investigational plan (PIP) in the early stages of the development of a new drug. In this PIP, a full description has to be given of the studies and of drug formulation in the paediatric population. If little information is available about the efficacy and safety of a drug, studies in children are only performed after more information has been obtained in the adult population to increase the safety of the paediatric study [10-12]. The main aims of introducing the paediatric regulation were to facilitate development and availability of medicines in children aged between 0 and 17 years, to improve the availability of information about medicines used in children, to ensure that the medicines are of high quality, can be administered in a safe and effective way and that paediatric studies are performed in an ethically correct way [10]. The reward for this effort is a 6-month supplementary production certificate for the pharmaceutical company.

Both for industry and for academic researchers, performing (PK-PD) studies in children in order to develop rational dosing schemes is very challenging because of ethical and practical issues. Unlike studies in healthy adults, research in healthy children is considered to be unethical, so all paediatric studies are performed in the vulnerable group of children suffering from a disease. In all clinical trials, informed consent has to be signed by the patient before he or she can be enrolled into a trial. In paediatric trials, this informed consent cannot be obtained by the patient that participates in the trial, and is therefore replaced by the consent of the parents or guardians. In older age groups, in addition to this consent, an assent is used in which the aim of the study is explained in age-appropriate language so that children can understand. $[1,4,13]$.

Apart from ethical issues, practical challenges also occur when performing studies in children. There are limitations to the number and volume of samples that can be obtained, resulting in infrequent sampling possibilities and the need for advanced drug assay techniques with improved sensitivity. Another complicating factor is the limited available number of subjects that suffer from the same disease. Finally, pharmacodynamic endpoints that measure the efficacy of the drug and that are validated for children may be lacking. All these factors call for highly advanced study designs and analysis techniques so that the burden for each child can be kept to a minimum while still addressing all the study objectives.

This article aims to inform clinical pharmacologists, paediatricians and pharmacists about population PK-PD modelling in paediatric drug research. Advanced statistical tools are discussed that can be used to develop rational dosing schemes based on the PK and PD of a drug in children, despite practical and ethical restrictions. Using these tools, covariates can be identified in order to define appropriate doses and dosing intervals based on the individual characteristics of each child with minimal burden to each patient. The article also describes how to evaluate the predictive performance of the models by different validation methods including a prospective clinical trial. Ultimately, the efforts result in an individualised dosing regimen based on the PK-PD relation throughout the paediatric age range.

\section{PK-PD in children}

Developmental changes in childhood can affect all PK processes from absorption until elimination as well as the pharmacodynamic effects. For example, in neonates intragastric $\mathrm{pH}$ is elevated $(>4)$, which may increase the bioavailability of acid-labile compounds (penicillin G) and decrease the bioavailability of weak acids (phenobarbital) when given orally [14]. Additionally, gastric emptying in neonates is delayed, which means that also the absorption 
of drugs, e.g. paracetamol is slower in neonates $[15,16]$. Other examples are changes in the metabolising enzyme capacity in children. Although most uridine $5^{\prime}$-diphosphate (UDP)-glucuronosyltransferases (UGTs) and P-450 cytochromes (CYPs) are expressed during the first week of life, the activity at birth in comparison with adults is often low, e.g. UGT2B7 activity at birth is around $10 \%$ of the adult level and different enzyme systems are known to mature at different rates [14, 17-20].

In addition, renal function and liver flow are influenced by physiological changes depending on age, e.g. the glomerular filtration rate (GFR) in $\mathrm{mL} / \mathrm{min} / 70 \mathrm{~kg}$ in fullterm neonates is $35 \%$ of the adult value, while $\mathrm{mL} / \mathrm{min} /$ $70 \mathrm{~kg}$ adult values are reached at approximately 1 year old [21]). When using units of $\mathrm{mL} / \mathrm{min} / 70 \mathrm{~kg}$, however, it should be realised that actual values of GFR in children are still very low compared with adult values because of the correction for differences in total body weight between adults and infants.

Furthermore, the body composition of children changes continuously, resulting in an age-dependent proportion of body water and fat, which influences the distribution of drugs. For example, the total amount of body water (80$90 \%$ of the body weight) is higher in neonates than in adults (55-60\%). Hydrophilic drugs like aminoglycosides have a larger volume of distribution in neonates, which can be explained by the larger amount of extra-cellular fluid ( $45 \%$ of the body weight) compared with adults $(20 \%)$ $[14,22]$.

In order to characterise the specific influence of developmental changes in childhood on the PK of a drug, concentration-time profiles are necessary, which require measurements of drug concentrations. For ethical reasons, in paediatric studies, discomfort, like pain and anxiety associated with venipuncture, must be restricted and practical issues limit the volume and amount of blood samples that can be obtained. Therefore, sensitive analysis techniques requiring only small blood samples should be used. While HPLC methods have been reported to require only $50 \mu \mathrm{L}$ of blood [23], more recently LC-MS methods can measure up to ten different drugs in volumes as low as $50-100 \mu \mathrm{L}$ [24]. Additionally, also alternative matrices such as saliva should be explored as a non-invasive, more child-friendly alternative to measure a drug concentration. An example in this respect is an LC-MS/MS method that was developed and validated for the measurement of busulphan in saliva [24]. Also, the use of a dried blood spot method, e.g. for tacrolimus, can facilitate the measurement of drugs in children [25]. Another method is capillary electrophoresis, which requires only a low sample volume for the quantification of drugs in biological fluids [26].

Changes between children and adults may also result from differences in the pharmacodynamics of a drug in children, e.g. by changes in the relative number and function of receptors. These age-related PD differences are until present rarely reported in the literature, but one of the few examples is the increased sensitivity to d-tubocurarine, an antagonist of nicotinic neuromuscular acetylcholine receptors, in neonates and infants compared with children and adults [27]. Other examples are the observed lower minimum alveolar concentration (MAC) of isoflurane in pre-term neonates compared with full-term neonates and older children [28, 29] and the different sensitivity to bronchodilators because of the lack of smooth muscles in the airways in neonates [30].

To study the PD of a drug in children, the use of a PD endpoint that has been validated for use in children is a prerequisite. An illustrative example is the measurement of pain in young children. Since they are not able to report their pain using a visual analogue scale, an observational scale has been developed. This comfort behavioural (COMFORT-B) scale was developed and validated for use in children under the age of 3 years [31]. The scale assesses six behavioural items: alertness, calmness, muscle tone, body movement, facial tension and crying (non-ventilated children) or respiratory response (ventilated children). All items range from 1 (no distress) to 5 (severe distress), resulting in a total score varying from 6 to 30. This validated scale can then be used as a PD endpoint for the development of PD models for pain and/or sedation in children of different ages [32-34].

The influence of covariates such as the developmental changes, disease status and genetics on the PK and PD of drugs in children is depicted in Fig. 1.

When both the PK and PD of a drug in children are characterised, the models developed can be used to derive rational dosing regimens with predictable efficacy and concentration profiles. An example of such a PK-PD model with a derived dosing regimen is an article published by Peeters et al. In this article both the PK and the PD were characterised in children, the latter with the use of the COMFORT-B scale as a pharmacodynamic endpoint [33]. Based on the model it was found that propofol clearance is

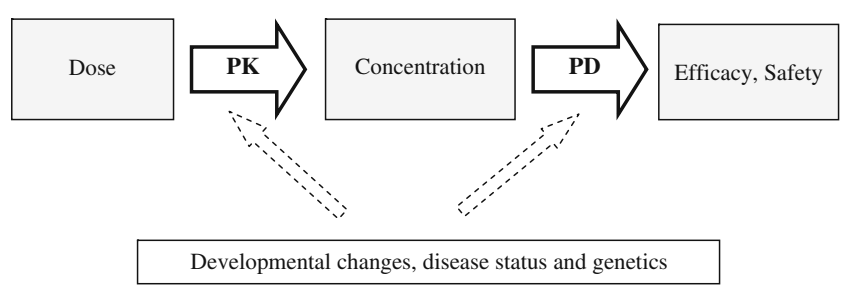

Fig. 1 Schematic representation of the relationship between dose and concentration (pharmacokinetics, PK) and between concentration and a pharmacological (side) effect (pharmacodynamics, PD). Important covariates that may affect both the PK and/or PD are body weight, age, disease status (e.g. critically ill versus healthy children) and genetics 
two times higher in non-ventilated children than in ventilated children and adults. For the PD, a model was derived in which an effect of propofol was characterised within a naturally occurring sleep pattern of children in the ICU. Both models (PK as well as PD) were used to simulate concentrations as well as the effects that could be expected using different dosing schemes (Fig. 2). As a result, based on this PK-PD model, a propofol dose of $30 \mathrm{mg} / \mathrm{h}$ was recommended for a child of $10 \mathrm{~kg}$, which will result in adequate COMFORT-B scales in the night following craniofacial surgery.

\section{Methods of analysing data: standard two-stage or population approach}

When concentration-time and concentration-effect datasets obtained in children are considered for analysis, two different methods can be applied: the standard two-stage approach and the population approach using non-linear mixed effect models [35-38]. When using the standard two-stage approach or classical approach, in a first step parameters are estimated in each individual based on individual concentration-time profiles (Fig. 3a). In a second step, these parameters are summarised by calculating the mean or median of the parameters and the variability between subjects (SE or IQR). A major drawback of this methodology is that it requires a relatively high number of samples in each individual patient (Fig. 3a), while each patient has to contribute roughly the same number of samples. Moreover, it is very difficult to distinguish between inter-individual (variability between subjects) and intra-individual or residual variability (variability within one subject, measurement error and model misspecification) and as a result inter-individual variability is often overestimated [39].

Since usually only a limited number of observations can be obtained in paediatric subjects, the population approach using non-linear mixed effect modelling to obtain PK and PD parameters is the preferred approach [37]. The population approach differs from the standard two-stage approach in the fact that the analysis is based on simultaneous analysis of all data of the entire population, while still taking into account that different observations come from different patients (Fig. 3b). Additionally, the population approach allows not only for the analysis of dense data, but also for sparse (limited number of observations per individual) and unbalanced data (unequal distribution of observations in various parts of the concentration-time profile in the individuals) or a combination of both. Finally, both the inter-individual and intra-individual variability are separately estimated in the dataset using this approach.

As a result of this methodology, when designing a paediatric study of which the data will be analysed using the population approach, it is advisable to collect samples at different times (or time windows) or to set alternating sampling schemes in subgroups of patients. This also means that (part of the) samples can be collected during routine clinical sampling. Consequently, the burden for the child who participates in the trial is reduced and the statistical power to develop a model describing the concentration-time or concentration-effect profile is not affected or improved.
Fig. 2 Simulation of propofol concentrations and response using COMFORT-B score versus time based on developed PK and PD models, after administration of different doses of propofol $(0,18,30$, and 36 $\mathrm{mg} / \mathrm{h}$ ) for a $10-\mathrm{kg}$ and a $5-\mathrm{kg}$ non-ventilated infant in the first night at the Intensive Care Unit following craniofacial surgery. Target COMFORT-B scores are between 12 and 14 preferably. Reproduced from [Peeters MY, Prins SA, Knibbe CA, DeJongh J, van Schaik RH, van Dijk M, et al. Propofol pharmacokinetics and pharmacodynamics for depth of sedation in nonventilated infants after major craniofacial surgery. Anesthesiology 2006 Mar;104(3):466-74.]

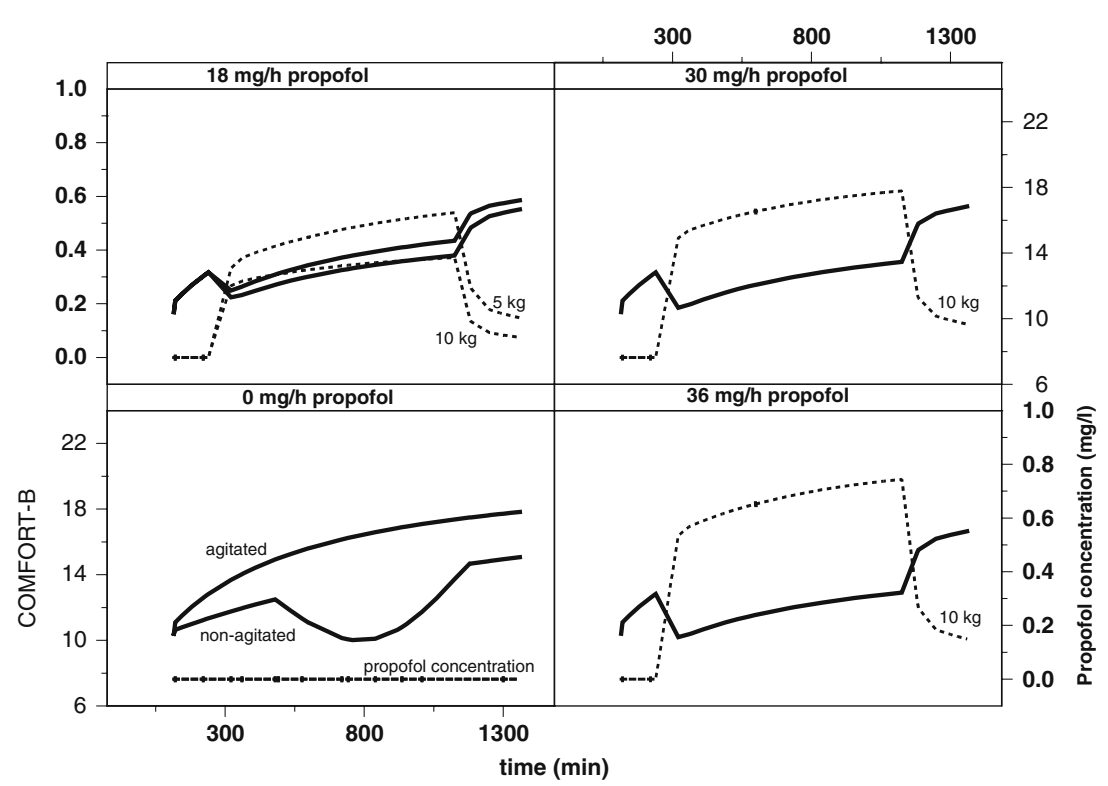



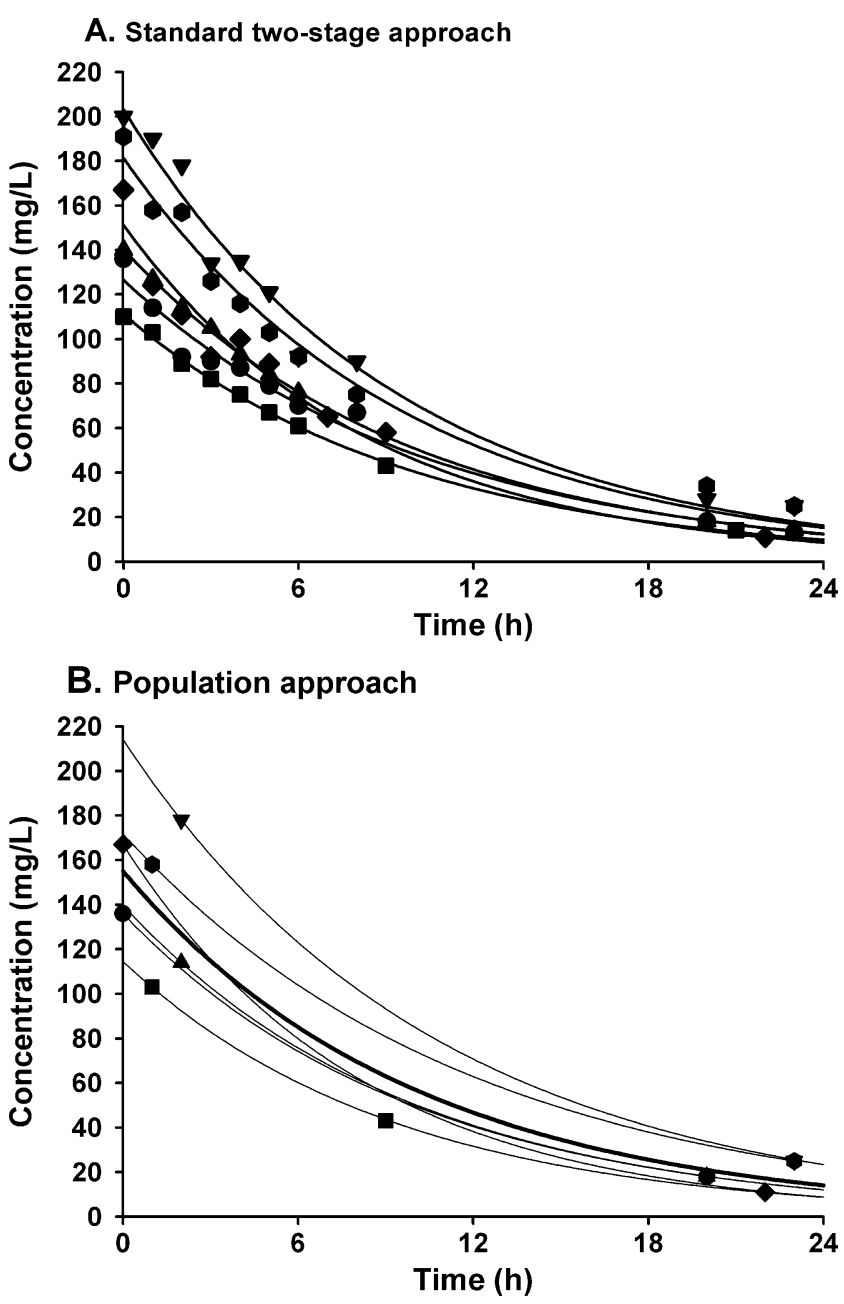

Fig. 3 Concentration-time profiles of the same study using two different approaches. In a the standard two-stage approach is applied to a rich dataset. $\mathbf{b}$ shows the population approach with mixed effect modelling applied to the same dataset using only two data points for each individual so that a sparse dataset is created. In $\mathbf{a}$, in each of the six individuals 10 samples are available. The different symbols correspond to different individuals. Each black line corresponds to a separate fit to the 10 data points of each individual. In $\mathbf{b}$, which uses the mixed effect modelling approach, two samples of the 10 per subject in a are used. The different symbols correspond to the six different individuals. The black line illustrates the concentration-time plot based on the population mean values of the PK parameters (PRED). The grey lines show the plots of the individual patients, which are based on the population mean values together with the measured concentrations of the specific individual (IPRED)

The term "mixed" in non-linear mixed effects modelling represents a mixture of fixed and random effects. For the fixed effects, a structural model describing the PK or PD is chosen (e.g. a two-compartment model for $\mathrm{PK}$ or an $\mathrm{E}_{\max }$ model for PD). The random effects quantify the variability that is not explained by the fixed effects. These random effects include inter-subject and intra-subject random variability (Fig. 4), which are both simultaneously and separately estimated. It is often assumed that the variability between subjects follows a normal distribution with a mean of zero and variance $\omega^{2}$. Equation 1 is used to describe the relationship between individual and population parameter estimates.

$\theta_{i}=\theta_{\text {mean }} \bullet e^{\eta i}$

where $\theta_{i}$ represents the parameter of the $i$ th subject, $\theta_{\text {mean }}$ the population mean, and $\eta \mathrm{i}$ the variability between subjects. The residual error is generally described using a proportional error (error is dependent on the concentration, which means a higher absolute error at higher concentrations (Eq. 2) or an additive error (constant for all observations (Eq. 3) or a combination of both. This means for the $j$ th observed concentration of the $I$ th individual the relation $\left(\mathrm{Y}_{\mathrm{ij}}\right)$ :

$Y_{i j}=c_{p r e d, i j} \bullet\left(1+\varepsilon_{i j}\right)$

$Y_{i j}=c_{p r e d, i j}+\varepsilon_{i j}$

where $c_{\text {pred }}$ is predicted concentration and $\varepsilon_{\mathrm{ij}}$ is a random variable with a mean of zero and a variance of $\sigma^{2}$.

In general, model building requires three different steps. First, a structural model (fixed effects) has to be designed, then a statistical sub-model (random effects) has to be developed and in the final step a covariate sub-model is identified.

The structural model describes the overall trend in the data. The choice of structural model (e.g. one-, two- or three-compartment model for $\mathrm{PK}$ and an $\mathrm{E}_{\max }$ model for PD) is to be based upon the best a priori information about the drug to be studied [40]. The structural model uses fixed effects parameters such as clearance and volume of distribution for $\mathrm{PK}$ or $\mathrm{E}_{\max }$ and $\mathrm{EC}_{50}$ for $\mathrm{PD}$. The population values for these parameters are called typical values (TV).

After selecting the structural model, the statistical submodel, which accounts for the inter-individual as well as the residual variability, is chosen and tested. Information on the inter- and intra-individual or residual variability is of clinical value, because it describes differences in clinical response between and within patients and may therefore provide guidance for rational dose adjustments. With the population approach, both these random effects are obtained, apart from estimates of both the population values (TV) and the individual values of $\mathrm{PK}$ and $\mathrm{PD}$ parameters (so-called post hoc parameter estimates).

In the final step the covariate sub-model is determined, which expresses relationships between covariates and parameters of the structural model (e.g. influence of body weight on volume of distribution or clearance). Covariates 


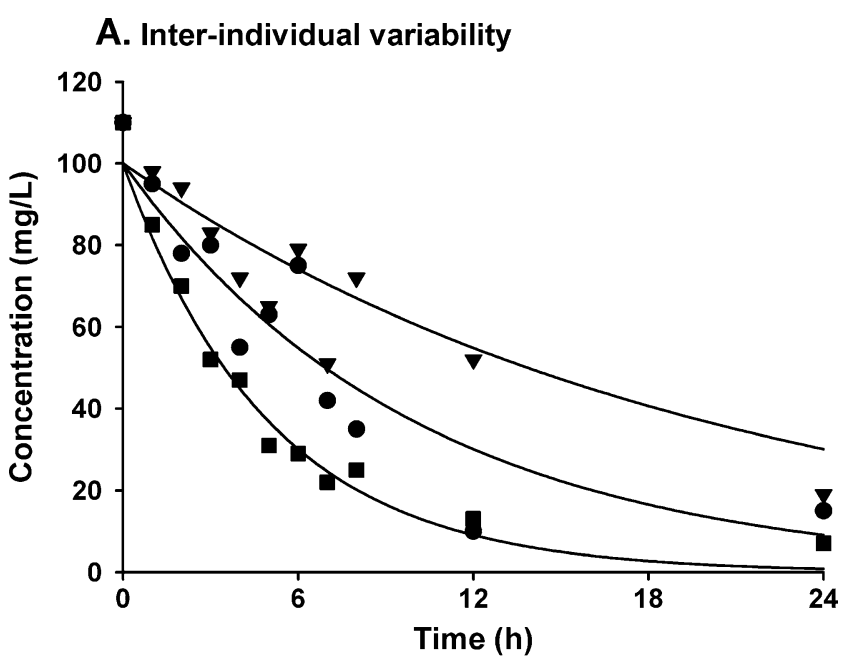

B. Intra-individual variability

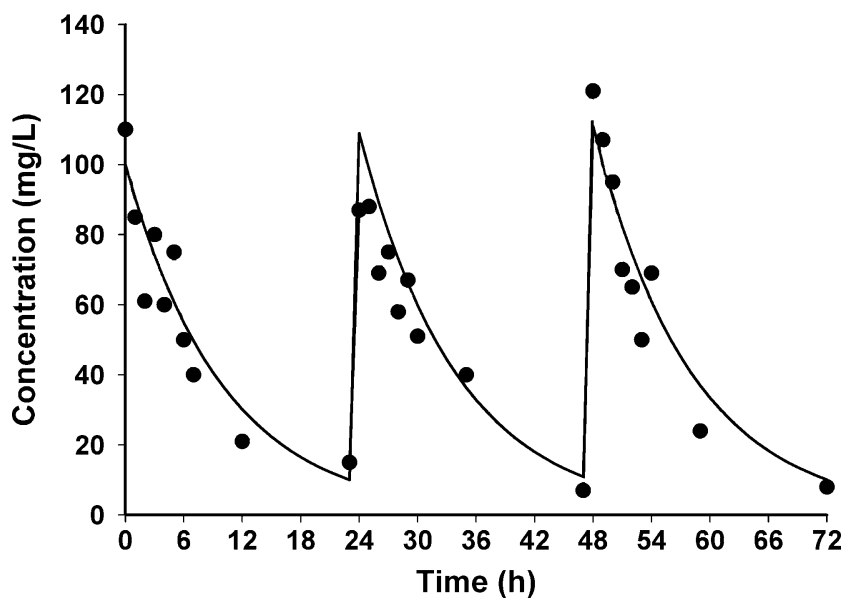

Fig. 4 In a, the inter-individual variability among three individuals who received the same dose is shown. b presents the intra-individual or residual variability by showing the concentration-time profile after repeated administration. Both these random variables are assumed to be normally distributed with a mean of zero and a variance of $\omega^{2}$ or $\sigma^{2}$ respectively

can be individual-specific (age, body weight, genetic profile, etc.) or time-varying (renal function, haemodynamic parameters, body temperature, etc.). The covariate analysis will be explained in more detail in the following section.

As these three models are inter-related, the choice of the structural (and statistical) model may affect the choice of the covariate model and vice versa. The process of finding a model that adequately describes the data is thus an elaborate task, where model checking/refining is performed in several steps. To assess model fit in relation to the observed concentrations or effect measures, scatter plots or the so-called goodness-of-fit plots are created (see Validation of the PK-PD models). Free software packages (Xpose, PSN, etc.) are available to generate these plots.
The most commonly used software package for model building, which is also supported by the European Medicines Agency (EMEA) is the non-linear mixed-effect modelling program NONMEM (GloboMax/ ICON, Ellicott City, MD, USA) [4, 41-43]. NONMEM estimates parameters (e.g. clearance, volume of distribution or $\mathrm{EC}_{50}$ ) via a maximum likelihood approach. This means that with the given data, the estimations of the parameters are those that occur with the highest probability. Alternative software packages that can be used are, for example, Monolix, WinNonMix, USC*PAC, which uses non-parametric maximum likelihood methods [44], or ADAPT, using maximum a posteriori (MAP) methods [45].

\section{Covariate analysis}

To determine the optimal dose based on the individual characteristics of a patient, a covariate analysis has to be performed [40, 46, 47]. The aim of the covariate analysis is to identify specific predictors (covariates) of PK and PD variability and can typically be studied in population models. Covariate analysis involves the modelling of the distribution of the individual parameter estimates as a function of covariates, which can be of demographic (e.g. age, body weight, gender), patho-physiological (e.g. renal or hepatic function) and genetic/environmental origin, and/ or be the result of the concomitant use of other drugs, which may influence the PK and/or PD. The identification of predictive covariates for variability provides the scientific basis for rational and individualised, patient-tailored dosing schemes.

The influence of developmental changes in childhood can be explored primarily by using size and/or age as covariates. Size (body weight) can be incorporated into the model using two different approaches. The first approach, the "allometric size approach", includes size a priori by using a body weight-based exponential equation with a fixed exponent of 0.75 for clearance and 1 for volume of distribution [48-52]. Once size is incorporated in the model using this fixed method, the influence of age is investigated, being the difference between the actual value of the PK parameter and the 0.75 allometric equation. When incorporating age as a covariate, different age descriptors may be used like postmenstrual age (PMA), gestational age (GA) or postnatal age (PNA) [53]. The choice of any of these age descriptors is based on the results of the systematic covariate analysis as described below [50, 54]. In the second approach, the "systematic covariate analysis", body weight is regarded as a covariate as any other, which means that the descriptive properties on the PK parameters are evaluated in a systematic covariate analysis as described below [55-57]. 
In a systematic analysis, when studying the influence of covariates, scatter plots and summary plots of individual parameter estimates and/or weighted residuals versus covariates are used to screen for appropriate covariates to include in the covariate sub-model. Additionally, these plots are used to explore the nature of the influence of the covariate (linear, exponential, allometric, subpopulations, etc.). Likely candidate covariates are then added to the model (forward inclusion). The influence of each covariate on the parameters is examined separately and compared with the simple model (no covariates). To assess whether the model with the covariate statistically improved the fit to the data, the difference between their objective function value, referred to as the $\log$-likelihood ratio, is calculated. This ratio is assumed to be Chi-squared distributed, which means that a reduction in objective function of 3.84 is considered to be significant $(P<0.05)[43,58]$. In addition, the reduction in objective function, goodness-of-fit plots of the simple model and covariate model are explored for diagnostic purposes. Furthermore, the confidence interval of the parameter estimates, the correlation matrix (indicates the relationship between two structural parameters) and visual improvement of the individual plots are used to evaluate the model. Finally, a superior model is expected to reduce the intersubject variance and/or the residual error terms. This procedure of covariate modelling implies that each covariate is only implemented if it can be fully justified by the data and the results of the statistic evaluations.

When two or more covariates are found to significantly improve the model, the covariate that reduced the objective function most is included in the model after which the other covariates are tested again for their significance. After all covariates that significantly improved the objective function are added to the simple model, a backward deletion is performed, which means that each covariate is removed from the full model, one at a time (the one that causes the smallest increase in objective function first). Retaining or removing the covariate is statistically tested by the use of the objective function (Chi-squared test) until each covariate has been tested.

In datasets containing sparse data, there may not be enough information to accurately estimate inter- and intraindividual variability. This causes the values of these parameters to shrink to 0 , resulting in individual parameter estimates that are closer to the population parameter estimates than they really are. This phenomenon is called shrinkage [59]. Shrinkage may cause individual predictions, individual parameter estimates and diagnostics based on them to be less reliable. It can also hide, falsely introduce or distort the shape of covariate relationships.

Shrinkage is the result of the properties of the data and is therefore difficult to avoid. One can only be aware of the presence of shrinkage, realise the influence it may have on the covariate analysis and use diagnostics other than those based on individual predictions or individual weighted residuals in the model building and model evaluation procedures.

\section{Validation of PK-PD models}

The objective of a PK or PK-PD modelling exercise is usually not just to describe the dataset of the sample of individuals that were studied. Generally, models are used to simulate which concentrations and/or effects and their variability can be expected when different doses are given to future patients. These simulations may therefore lead to optimised dosing recommendations or to optimisation of new studies for the entire population to which the sample of individuals belongs. It is often said that "all models are wrong, but some are useful" [60]. In order to define whether a model is useful and valid for clinical and trial simulations, thorough evaluation and validation of the model is necessary. Although validations of PK models are only performed in $17 \%$ of the published paediatric studies [4] and in $28 \%$ of the adult studies [61], proper model validations are an essential step in model building. For this purpose, different evaluation and validation methods are available. As described before [62], a proper validation and evaluation procedure includes an internal model evaluation followed by an external evaluation and a prospective clinical study.

The first evaluation method is the basic internal model validation used to assess whether the model is able to describe the learning dataset (the dataset used to develop the model) accurately and without bias. This evaluation should actually be considered the final stage of the model building procedure. Subsequently, in the external evaluation, it is assessed whether the model is able to describe one or more external datasets (the datasets other than the one used to develop the model) adequately. Alternatively, if a dataset is sufficiently large the original dataset may be split in two so that the model is developed using one part (about two thirds) of the dataset and evaluated externally using the other part (one third) of the dataset. In paediatric studies, it is then especially important to stratify the data correctly and ascertain that all age groups are represented in equal proportions in both datasets.

Various techniques are available for the validation and evaluation of population PK and PK-PD models (both for internal and external validation procedures).

\section{Basic goodness-of-fit plots}

1. Individual predicted vs observed concentrations

2. Population predicted vs observed

3. (Conditional) weighted residual vs time

4. (Conditional) weighted residuals vs dependent variable 
Weighted residuals (WRES) and conditional weighted residuals (CWRES) are calculated as follows:

$W R E S=\frac{\vec{y}_{i}-E_{F O}\left(\vec{y}_{i}\right)}{\sqrt{\operatorname{Cov}_{F O}\left(\vec{y}_{i}\right)}}$

CWRES $=\frac{\vec{y}_{i}-E_{F O C E}\left(\vec{y}_{i}\right)}{\sqrt{\operatorname{Cov}_{F O C E}\left(\vec{y}_{i}\right)}}$

where $\vec{y}_{i}$ is the vector of the measurements, $E\left(\vec{y}_{i}\right)$ is the expectation of the data and $\operatorname{Cov}\left(\vec{y}_{i}\right)$ is the covariance matrix of the data [63].

These plots are used in model building, but can also be used to ascertain that there is no trend or bias in the model predictions of the final model. Furthermore, these plots can be used for both the internal and external evaluation of the model.

Bootstrap analysis In a bootstrap analysis new datasets are generated by resampling from the original dataset and it is therefore an internal validation of the model. The new datasets are subsequently refitted to the original model, yielding mean values and standard errors for every model parameter.

A bootstrap analysis provides information on the stability of the model and its dependence on specific individuals in the learning dataset. With the freely available PSN or Wings for NONMEM software packages an automated bootstrap analysis can be performed.

Visual predictive check In a visual predictive check (VPC) [64] a PK or PD profile is simulated a 100 to 1,000 times and lines for the median values and their $90 \%$ prediction interval are plotted in a graph. The observed values in the internal or external dataset are subsequently plotted on top of this. It can then be visually checked whether $90 \%$ of the observations are within the indicated prediction interval and whether there is no bias in the observations compared with the prediction interval. In Fig. 5, two examples of a VPC are given, showing when a model does not work and when a model does work on the same data.

The VPC is a simulation-based diagnostic that can be used when the PK or PD profiles for all individuals in the dataset are similar and it allows for easy interpretation of the result. For this diagnostic tool, there are no statistical tests and all evaluations are based on visual assessment. When the individual profiles are expected to deviate largely from one another because there is for instance a large variability in the time and amount of dose administrated, or when there are many covariates, the use of this diagnostic becomes more difficult.
Normalised prediction distribution error Another simulationbased diagnostic that can be used for both internal and external validations is the normalised prediction distribution error (NPDE) [65]. An example of an NPDE previously published is shown in Fig. 6 [55]. This method yields information on how accurate the model predicts the median value of the observations and the variability within them. The interpretation of this diagnostic is less straightforward than for the VPC, but the advantage of this method is that it can be used when the variability in dosing regimen (both in time, amounts and rates) is high or when there is a large
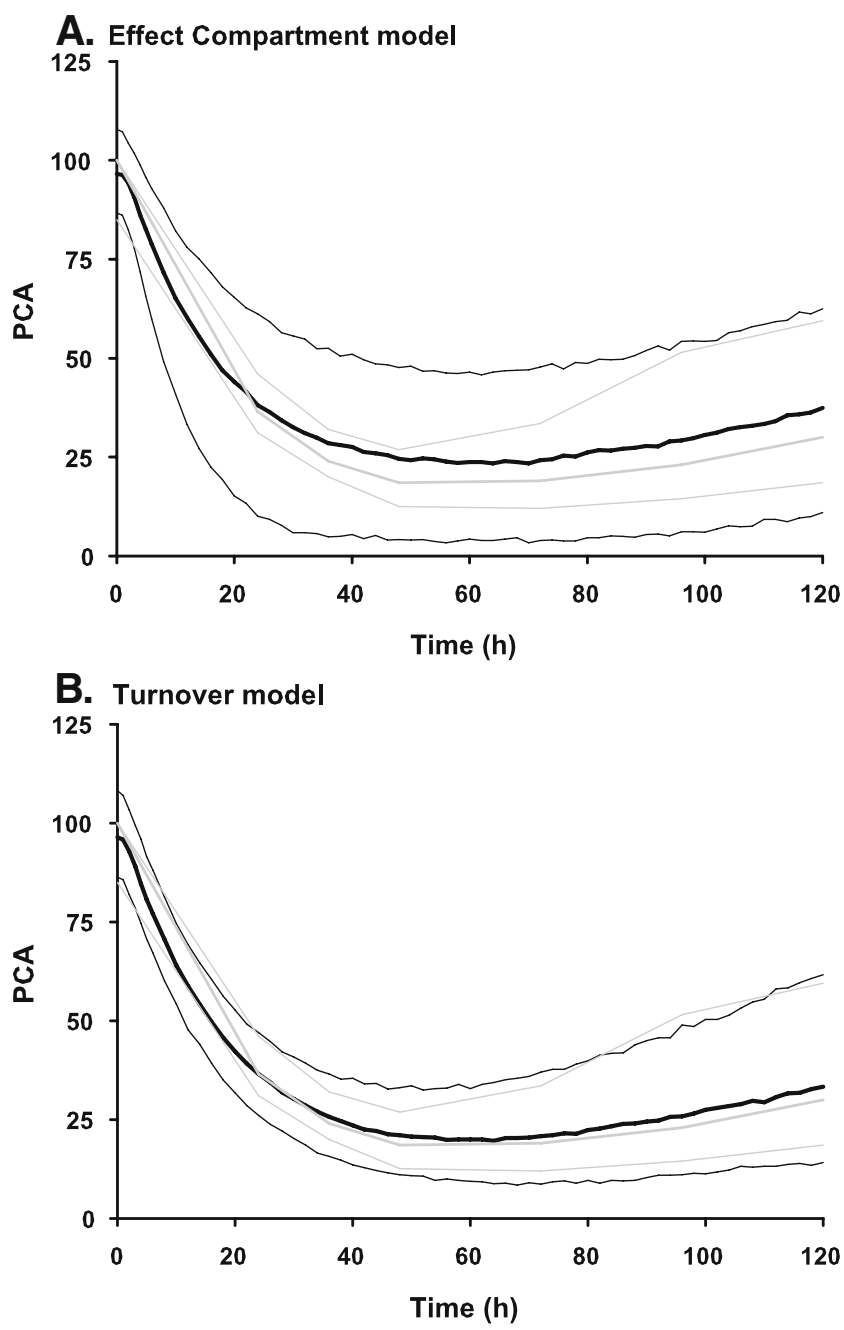

Fig. 5 Two examples of a visual predictive check (VPC) are illustrated based on the same dataset (warfarin concentrations and prothrombin complex activity [PCA]) using two different models. In a the VPC of the effect compartment is shown, while in $\mathbf{b}$ the VPC of the turnover model is demonstrated. Both real (grey) and simulated (black) data are displayed with mean (thick lines) and 95\% intervals (thin lines). Based on both graphics, the turnover model is the most appropriate model since real and simulated lines are in good agreement. Reproduced from [Karlsson, M. O. and N. H. G. Holford (2008). "A Tutorial on Visual Predictive Checks." PAGE 17 (2008) Abstr 1434 (www.page-meeting.org/?abstract=1434)] 


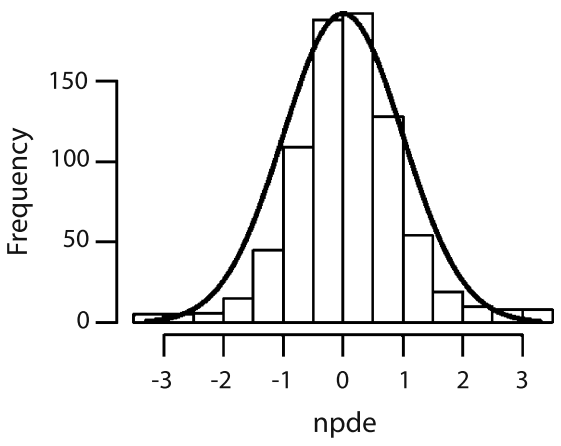

Mean : $0.074^{*}$

Variance : $0.90^{*}$

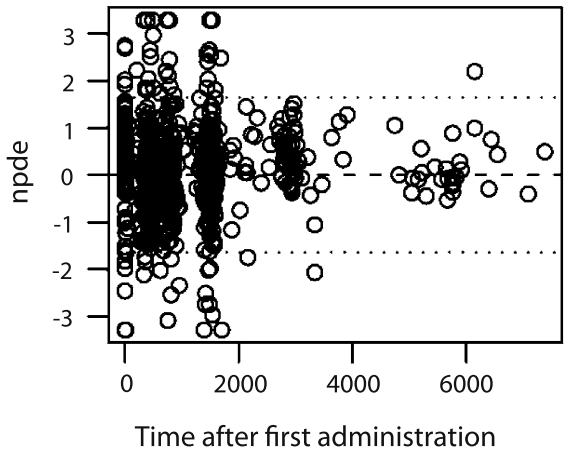

Time after first administration

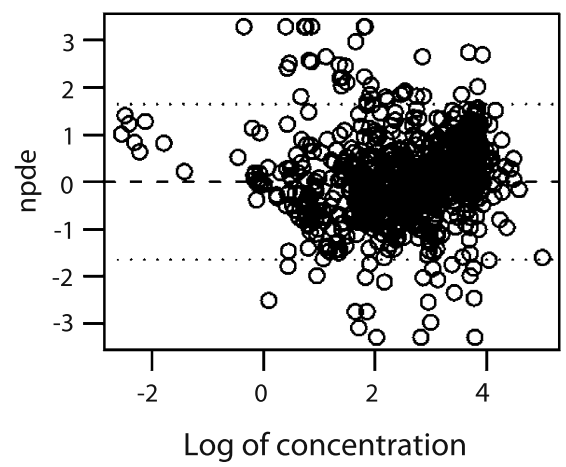

Log of concentration
Fig. 6 Example of a normalised prediction distribution error (NPDE) analysis, which shows the NPDE distributions for morphine. The normal distribution is represented by the solid line. The values for the mean and standard deviation of the observed NPDE distribution are given below the histogram, with an asterisk indicating a significant difference of a mean of 0 and a variance of 1 at the $P<0.05$ level, as determined by the Wilcoxon signed rank test and Fisher's test for variance. The distribution of NPDE vs time after the first dose and

number of covariates in the model. This can for instance be the case for data obtained during routine paediatric clinical practice. Software (e.g. NPDE add-on package for R) [66] to perform this analysis is freely available. For the NPDE, besides visual evaluation of the plots, statistical tests are available. These statistical tests are, however, reported to be highly sensitive and powerful, so that decisions for the model should primarily be based on visual assessments. An example is the statistically significant deviation of zero of the mean value because of the large number of data, while the actual deviation is small (e.g. 0.074) and not of clinical relevance.

If the model performed well in both evaluation procedures, the dosing algorithm that results from the PK-PD model needs to be tested and challenged in a prospective (clinical) trial. If the predictive performance of the model is corroborated by the trial it can be used with confidence in clinical practice.
NPDE vs the log of the concentrations are also shown. The dotted lines represent the $90 \%$ distribution of the NPDE. Reproduced from [Knibbe CA, Krekels EH, van den Anker JN, DeJongh J, Santen GW, van Dijk M, et al. Morphine glucuronidation in preterm neonates, infants and children younger than 3 years. Clin Pharmacokinet 2009;48(6):371-85] with permission from Wolters Kluwer Health | Adis (C Adis Data Information BV [2006]). All rights reserved

\section{Optimal design of paediatric studies}

When new population PK-PD studies are performed, it is important to design these studies in the most efficient manner possible to obtain maximum information about the PK and PD parameters so that they can be determined with the highest precision [51, 67]. When designing PK-PD studies in paediatrics certain factors need to be taken into account, e.g. the age range of the paediatric group, the therapeutic index, the possibility of collecting blood samples, the availability of validated PD endpoints for children and the availability of sensitive analytical methods.

When optimising a PK or PK-PD study design, using literature data from adults or children of different age ranges or possibly in vitro or pre-clinical data, a concentration-time or effect-time profile for a study can be simulated. This can help to identify possible shortcomings in the design or to perform a power analysis. Alternatively,

Table 1 Factors influencing the required number of patients and/or samples per patient

\begin{tabular}{ll}
\hline Factor & Number of patients/samples \\
\hline Study of PK only & Relatively small number of patients/samples \\
Study of PK-PD relationship & Relatively high number of patients/samples \\
Even distribution of covariates (age, body weight) & $\downarrow$ Number of patients/samples \\
$\uparrow$ Number of changes in dose & $\uparrow /$ - Number of patients/samples (depending on other aspects of the study \\
& design) \\
$\uparrow$ Number of samples/child & $\downarrow$ Number of patients \\
Use of optimal sampling strategies & $\downarrow$ Number of patients/samples \\
Different sampling windows (e.g. two or three sampling & $\downarrow$ Number of patients/samples \\
schemes) &
\end{tabular}


software packages are available (WINPOPT [68], PopED [67] and PFIM [69]) that can help to identify the optimal number and time points of observations in a study based on the prior information on a drug [70]. To determine the appropriate sample size certain factors, which are summarised in Table 1, need to be taken into account. Each of these factors can influence the required number of patients and/or samples in a positive or negative way. In a study by Peeters et al. [32] only 24 patients (aged between 3 and 24 months) were required to determine both the PK and PD, since rich sampling was performed (a median of 11 samples per child) and no covariates were found in the relatively homogeneous population. This is in contrast to a study performed by Knibbe et al. [55], in which 250 children were included. This higher number was required because in addition to the large dispersion in age from (pre-term) neonates up to toddlers of 3 years of age, only 1 to 4 samples were available for each subject. Moreover, infusion rates and additional bolus doses varied for each child during the study to obtain the desired analgesic effect. In another example [71], only 6 patients (aged between 1 and 5 years) were required, in which 7 samples per patient were collected. This lower number of patients $(n=6)$ compared with the study by Peeters et al. $(n=24)$ can be explained because there often exists a lower variability in PK than in $\mathrm{PD}$, which results in a lower number of patients required (Table 1).

\section{Conclusions and perspectives}

In view of the European Regulation, which came into force in 2007, it now seems time to use the progress that has been made in the field of integrated PK-PD modelling [72] to develop rational and individualised dosing schemes for children. Because of the possibility of analysing sparse and unbalanced datasets, thereby minimising the burden for each child, population PK-PD modelling and simulation using non-linear mixed effect modelling has become the preferred tool to develop effective and safe dosing regimens for children. Specifically in paediatrics, where developmental changes have to be taken into account, which may influence the PK and/or the PD of the drugs, this advanced statistical tool is of critical value.

Before dosing regimens can be tested in clinical practice, proper validations of the models should be performed, for which adequate tools have recently been developed. Besides internal and external validation, prospective clinical trials, which allow for the evaluation of the model-based dosing regimens, are needed, not only to adjust the proposed dosing regimen, but also to convince paediatricians to use the information that has been generated using these modelling exercises.
Furthermore, one of the future goals may be to explore the possibilities of cross-validation of the models, in which the reported influences of developmental changes on a certain PK or PD parameter of one drug are evaluated for use in another drug that goes through the same metabolic route or shares the same mechanism of action. In this respect, physiologically based pharmacokinetic (PBPK) models are needed. PBPK models consider the physiological and biochemical processes by using in vitro data to describe the PK of drugs [73, 74]. The combination of these two approaches may use the information that is already available in an optimal way in defining effective and safe dosing regimens for each individual patient.

In conclusion, analyses of paediatric data using population PK-PD modelling and covariate analysis will result in individualised dosing regimens for children of different ages, body weights and genetic backgrounds. Thus, population PK-PD modelling constitutes an innovative approach to the study of drug effects in this very special patient population, which is otherwise difficult to study.

Acknowledgements The clinical research of K. Allegaert is supported by the Fund for Scientific Research, Flanders (Belgium) (FWO Vlaanderen) by a Fundamental Clinical Investigatorship (1800209 N). The clinical research of C.A.J. Knibbe is supported by the Innovational Research Incentives Scheme (Veni grant, July 2006) of the Dutch Organisation for Scientific Research (NWO). The research of E.H.J. Krekels is supported by the Top Institute Pharma project number D2-104. The authors would like to thank Professor Nick Holford and Professor Mats Karlsson for providing figure 5, Dr. Rifka Peeters for providing figure 2 and Pieter De Cock for critically reading the manuscript.

Open Access This article is distributed under the terms of the Creative Commons Attribution Noncommercial License which permits any noncommercial use, distribution, and reproduction in any medium, provided the original author(s) and source are credited.

\section{References}

1. Ernest TB, Elder DP, Martini LG et al (2007) Developing paediatric medicines: identifying the needs and recognizing the challenges. J Pharm Pharmacol 59(8):1043-1055

2. Jong GW, Vulto AG, de Hoog M et al (2001) A survey of the use of off-label and unlicensed drugs in a Dutch children's hospital. Pediatrics 108(5):1089-1093

3. Conroy S, Choonara I, Impicciatore P et al (2000) Survey of unlicensed and off label drug use in paediatric wards in European countries. European Network for Drug Investigation in Children. BMJ 320(7227):79-82

4. Tod M, Jullien V, Pons G (2008) Facilitation of drug evaluation in children by population methods and modelling. Clin Pharmacokinet 47(4):231-243

5. Bray RJ (1998) Propofol infusion syndrome in children. Paediatr Anaesth 8(6):491-499

6. Parke TJ, Stevens JE, Rice AS et al (1992) Metabolic acidosis and fatal myocardial failure after propofol infusion in children: five case reports. BMJ 305(6854):613-616 
7. Sutherland JM (1959) Fatal cardiovascular collapse of infants receiving large amounts of chloramphenicol. AMA J Dis Child 97 (6):761-767

8. McIntyre J, Choonara I (2004) Drug toxicity in the neonate. Biol Neonate 86(4):218-221

9. Nederland Kenniscentrum Farmacotherapie bij Kinderen. http:// www.kinderformularium.nl. Accessed on 29 June 2009

10. European Medicines Agency (EMEA): Medicines for children. http://www.emea.europa.eu/htms/human/paediatrics/introduction. $\mathrm{htm}$. Accessed on 17 June 2009

11. Dunne J (2007) The European Regulation on medicines for paediatric use. Paediatr Respir Rev 8(2):177-183

12. Consultant PR (2006) EU paediatric regulation for testing medicines in children. http://www.child-medicines-research-info. com/paediatric-research-consultancy/eu-paediatric-regulation.asp. Accessed on 23 June 2009

13. Saint Raymond A, Brasseur D (2005) Development of medicines for children in Europe: ethical implications. Paediatr Respir Rev 6 (1):45-51

14. Kearns GL, Abdel-Rahman SM, Alander SW et al (2003) Developmental pharmacology - drug disposition, action, and therapy in infants and children. N Engl J Med 349(12):1157-1167

15. Grand RJ, Watkins JB, Torti FM (1976) Development of the human gastrointestinal tract. A review. Gastroenterology 70(5 PT.1):790-810

16. Anderson BJ, van Lingen RA, Hansen TG et al (2002) Acetaminophen developmental pharmacokinetics in premature neonates and infants: a pooled population analysis. Anesthesiology 96(6):1336-1345

17. Hines RN, McCarver DG (2002) The ontogeny of human drugmetabolizing enzymes: phase I oxidative enzymes. J Pharmacol Exp Ther 300(2):355-360

18. De Wildt SN, Kearns GL, Leeder JS et al (1999) Glucuronidation in humans. Pharmacogenetic and developmental aspects. Clin Pharmacokinet 36(6):439-452

19. Leeder JS (2004) Translating pharmacogenetics and pharmacogenomics into drug development for clinical pediatrics and beyond. Drug Discov Today 9(13):567-573

20. Krekels EH, van den Anker JN, Baiardi P et al (2007) Pharmacogenetics and paediatric drug development: issues and consequences to labelling and dosing recommendations. Expert Opin Pharmacother 8(12):1787-1799

21. Rhodin MM, Anderson BJ, Peters AM et al (2009) Human renal function maturation: a quantitative description using weight and postmenstrual age. Pediatr Nephrol 24(1):67-76

22. Bartelink IH, Rademaker CM, Schobben AF et al (2006) Guidelines on paediatric dosing on the basis of developmental physiology and pharmacokinetic considerations. Clin Pharmacokinet 45(11):1077-1097

23. Knibbe CA, Koster VS, Deneer VH et al (1998) Determination of propofol in low-volume samples by high-performance liquid chromatography with fluorescence detection. J Chromatogr B Biomed Sci Appl 706(2):305-310

24. Rauh M, Stachel D, Kuhlen M et al (2006) Quantification of busulfan in saliva and plasma in haematopoietic stem cell transplantation in children: validation of liquid chromatography tandem mass spectrometry method. Clin Pharmacokinet 45(3):305-316

25. Edelbroek PM, van der Heijden J, Stolk LM (2009) Dried blood spot methods in therapeutic drug monitoring: methods, assays, and pitfalls. Ther Drug Monit 31(3):327-336

26. Jabeen R, Payne D, Wiktorowicz J et al (2006) Capillary electrophoresis and the clinical laboratory. Electrophoresis 27 (12):2413-2438

27. Fisher DM, O'Keeffe C, Stanski DR et al (1982) Pharmacokinetics and pharmacodynamics of d-tubocurarine in infants, children, and adults. Anesthesiology 57(3):203-208
28. LeDez KM, Lerman J (1987) The minimum alveolar concentration (MAC) of isoflurane in preterm neonates. Anesthesiology 67 (3):301-307

29. Blusse van Oud-Alblas HJ, Peeters M, Brill MJE, Knibbe CAJ, Klein J, Tibboel D, Weber F (2009) The pharmacodynamics of isoflurane in children using Bispectral index and composite auditory evoked potentials. www.page-meeting.org/ ?abstract $=1576$

30. Stephenson T (2005) How children's responses to drugs differ from adults. Br J Clin Pharmacol 59(6):670-673

31. Van Dijk M, de Boer JB, Koot HM et al (2000) The reliability and validity of the COMFORT scale as a postoperative pain instrument in 0 to 3-year-old infants. Pain 84(2-3):367-377

32. Peeters MY, Prins SA, Knibbe CA et al (2006) Pharmacokinetics and pharmacodynamics of midazolam and metabolites in nonventilated infants after craniofacial surgery. Anesthesiology 105 (6): 1135-1146

33. Peeters MY, Prins SA, Knibbe CA et al (2006) Propofol pharmacokinetics and pharmacodynamics for depth of sedation in nonventilated infants after major craniofacial surgery. Anesthesiology 104(3):466-474

34. Van Dijk M, Peters JW, van Deventer P et al (2005) The COMFORT Behavior Scale: a tool for assessing pain and sedation in infants. Am J Nurs 105(1):33-36

35. Jonsson EN, Karlsson MO (1999) Xpose-an S-PLUS based population pharmacokinetic/pharmacodynamic model building aid for NONMEM. Comput Methods Programs Biomed 58(1):5164

36. FDA C (1999) Guidance for industry. Population pharmacokinetics. http://www.fda.gov/downloads/Drugs/GuidanceCompliance RegulatoryInformation/Guidances/ucm072137.pdf

37. FDA C (1998) Guidance for industry. General considerations for pediatric pharmacokinetic studies for drugs and biological products (Draft Guidance). http://www.fda.gov/downloads/Drugs/Guidance ComplianceRegulatoryInformation/Guidances/ucm072114.pdf. Accessed on 22 June 2009

38. Beal SL, Sheiner LB (1982) Estimating population kinetics. Crit Rev Biomed Eng 8(3):195-222

39. Ette EI, Williams PJ, Lane JR (2004) Population pharmacokinetics III: design, analysis, and application of population pharmacokinetic studies. Ann Pharmacother 38(12):2136-2144

40. Wade JR, Beal SL, Sambol NC (1994) Interaction between structural, statistical, and covariate models in population pharmacokinetic analysis. J Pharmacokinet Biopharm 22(2):165-177

41. Aarons L (1999) Software for population pharmacokinetics and pharmacodynamics. Clin Pharmacokinet 36(4):255-264

42. Beal SL, Broeckman AJ (2006) NONMEM Users guides. Icon Development Solutions, Ellicott City, MD

43. Beal SL Broeckman AJ (1994) NONMEM Users Guide. V. University of California at San Francisco, San Francisco

44. Jelliffe RSA, Van Guilder M, et al. (1995) User manual for version 10.7 of the USC*PAC Collection of PC Programs, USC Laboratory of Applied Pharmacokinetics. USC School of Medicine, Los Angeles

45. Bustad A, Terziivanov D, Leary R et al (2006) Parametric and nonparametric population methods: their comparative performance in analysing a clinical dataset and two Monte Carlo simulation studies. Clin Pharmacokinet 45(4):365-383

46. Mentre F, Mallet A (1994) Handling covariates in population pharmacokinetics. Int J Biomed Comput 36(1-2):25-33

47. Mandema JW, Verotta D, Sheiner LB (1992) Building population pharmacokinetic-pharmacodynamic models. I. Models for covariate effects. J Pharmacokinet Biopharm 20(5):511-528

48. Anderson BJ, Holford NH (2008) Mechanism-based concepts of size and maturity in pharmacokinetics. Annu Rev Pharmacol Toxicol 48:303-332 
49. Anderson BJ, Allegaert K, Holford NH (2006) Population clinical pharmacology of children: general principles. Eur J Pediatr 165 (11):741-746

50. Anderson BJ, Allegaert K, Holford NH (2006) Population clinical pharmacology of children: modelling covariate effects. Eur $\mathrm{J}$ Pediatr 165(12):819-829

51. Meibohm B, Laer S, Panetta JC et al (2005) Population pharmacokinetic studies in pediatrics: issues in design and analysis. AAPS J 7(2):E475-E487

52. Anderson BJ, Holford NH (2009) Mechanistic basis of using body size and maturation to predict clearance in humans. Drug Metab Pharmacokinet 24(1):25-36

53. Engle WA (2004) Age terminology during the perinatal period. Pediatrics 114(5):1362-1364

54. Allegaert K, Anderson BJ, Cossey V et al (2006) Limited predictability of amikacin clearance in extreme premature neonates at birth. Br J Clin Pharmacol 61(1):39-48

55. Knibbe CA, Krekels EH, van den Anker JN et al (2009) Morphine glucuronidation in preterm neonates, infants and children younger than 3 years. Clin Pharmacokinet 48(6):371-385

56. Mahmood I (2007) Prediction of drug clearance in children: impact of allometric exponents, body weight, and age. Ther Drug Monit 29(3):271-278

57. Mahmood I (2009) Application of fixed exponent 0.75 to the prediction of human drug clearance: an inaccurate and misleading concept. Drug Metabol Drug Interact 24(1):57-81

58. Sheiner LB, Ludden TM (1992) Population pharmacokinetics/ dynamics. Annu Rev Pharmacol Toxicol 32:185-209

59. Karlsson MO, Savic RM (2007) Diagnosing model diagnostics. Clin Pharmacol Ther 82(1):17-20

60. Box G (1979) Robustness in the strategy of scientific model building. In: Launer RL, Wilkinson GN (eds) Robustness in statistics. Academic Press, New York, p 202

61. Brendel K, Dartois C, Comets E et al (2007) Are population pharmacokinetic and/or pharmacodynamic models adequately evaluated? A survey of the literature from 2002 to 2004. Clin Pharmacokinet 46(3):221-234

62. Ince I, de Wildt SN, Tibboel D et al (2009) Tailor-made drug treatment for children: creation of an infrastructure for data- sharing and population PK-PD modeling. Drug Discov Today 14 (5-6):316-320

63. Hooker AC, Staatz CE, Karlsson MO (2007) Conditional weighted residuals (CWRES): a model diagnostic for the FOCE method. Pharm Res 24(12):2187-2197

64. Fang L, Holford NH, Hinkle G et al (2007) Population pharmacokinetics of humanized monoclonal antibody $\mathrm{HuCC} 49 \mathrm{deltaCH} 2$ and murine antibody CC49 in colorectal cancer patients. J Clin Pharmacol 47(2):227-237

65. Brendel K, Comets E, Laffont C et al (2006) Metrics for external model evaluation with an application to the population pharmacokinetics of gliclazide. Pharm Res 23(9):20362049

66. Comets E, Brendel K, Mentre F (2008) Computing normalised prediction distribution errors to evaluate nonlinear mixed-effect models: the npde add-on package for R. Comput Methods Programs Biomed 90(2):154-166

67. Foracchia M, Hooker A, Vicini P et al (2004) POPED, a software for optimal experiment design in population kinetics. Comput Methods Programs Biomed 74(1):29-46

68. Duffull SB, Eccleston J, Kimco H (2008) WinPOPT user guide Version 1.2. School of Pharmacy, University of Otago, Otago, New Zealand

69. Retout S, Mentre F (2003) Optimization of individual and population designs using Splus. J Pharmacokinet Pharmacodyn $30(6): 417-443$

70. Duffull SB (2001) Design of clinical pharmacology trials. Clin Exp Pharmacol Physiol 28(11):905-912

71. Knibbe CA, Melenhorst-de Jong G, Mestrom M et al (2002) Pharmacokinetics and effects of propofol $6 \%$ for short-term sedation in paediatric patients following cardiac surgery. $\mathrm{Br} \mathrm{J}$ Clin Pharmacol 54(4):415-422

72. Breimer DD (2008) PK/PD modelling and beyond: impact on drug development. Pharm Res 25(12):2720-2722

73. Alcorn J, McNamara PJ (2008) Using ontogeny information to build predictive models for drug elimination. Drug Discov Today 13(11-12):507-512

74. Johnson TN (2005) Modelling approaches to dose estimation in children. Br J Clin Pharmacol 59(6):663-669 